\title{
Solitonlike solutions of the generalized discrete nonlinear Schrödinger equation
}

\author{
Rasmussen, Kim; Henning, D.; Gabriel, H.; Bülow, A.
}

Published in:

Physical Review E. Statistical, Nonlinear, and Soft Matter Physics

Link to article, DOI:

10.1103/PhysRevE.54.5788

Publication date:

1996

Document Version

Publisher's PDF, also known as Version of record

Link back to DTU Orbit

Citation (APA):

Rasmussen, K., Henning, D., Gabriel, H., \& Bülow, A. (1996). Solitonlike solutions of the generalized discrete nonlinear Schrödinger equation. Physical Review E. Statistical, Nonlinear, and Soft Matter Physics, 54(5), 57885801. https://doi.org/10.1103/PhysRevE.54.5788

\section{General rights}

Copyright and moral rights for the publications made accessible in the public portal are retained by the authors and/or other copyright owners and it is a condition of accessing publications that users recognise and abide by the legal requirements associated with these rights.

- Users may download and print one copy of any publication from the public portal for the purpose of private study or research.

- You may not further distribute the material or use it for any profit-making activity or commercial gain

- You may freely distribute the URL identifying the publication in the public portal 


\title{
Solitonlike solutions of the generalized discrete nonlinear Schrödinger equation
}

\author{
D. Hennig, ${ }^{1}$ K. Ф. Rasmussen, ${ }^{1,2}$ H. Gabriel, ${ }^{1}$ and A. Bülow ${ }^{1}$ \\ ${ }^{1}$ Freie Universität Berlin, Fachbereich Physik, Institut für Theoretische Physik Arnimallee 14, 14195 Berlin, Germany \\ ${ }^{2}$ Institute of Mathematical Modelling, Technical University of Denmark, Anker Engelundsvej, DK-2800 Lyngby, Denmark
}

(Received 14 June 1996)

\begin{abstract}
We investigate the solution properties of a generalized discrete nonlinear Schrödinger equation describing a nonlinear lattice chain. The generalized equation interpolates between the integrable discrete Ablowitz-Ladik equation and the nonintegrable discrete Schrödinger equation. Special interest is paid to the creation of stationary localized solutions called breathers. To tackle this problem we apply a map approach and illuminate the linkage of homoclinic and heteroclinic map orbits with localized lattice solutions. The homoclinic and heteroclinic orbits correspond to exact nonlinear solitonlike eigenstates of the lattice. Normal forms and the Melnikov method are used for analytical determinations of homoclinic orbits. Nonintegrability of the map leads to soliton pinning on the lattice. The soliton pinning energy is calculated and it is shown that it can be tuned by varying the ratio of the nonintegrability parameter versus the integrability parameter. The heteroclinic map orbit is derived on the basis of a variational principle. Finally, we use homoclinic and heteroclinic orbits as initial conditions to excite designed stationary localized solutions of desired width in the dynamics of the discrete nonlinear Schrödinger equation. In this way we are able to construct coherent solitonlike structures of profile determined by the map parameters. [S1063-651X(96)10211-7]

PACS number(s): 03.40.Kf, 63.20.Pw, 63.20.Ry
\end{abstract}

\section{INTRODUCTION}

In recent studies of the nonlinear dynamics of lattice systems consisting of coupled oscillators attention was paid to time-periodic and spatially localized excitations [1-10]. A prototype of such a nonlinear lattice is represented by the nonlinear Schrödinger equation (DNLS), the physical importance of which is well documented in a series of publications [11-18]. The DNLS arises also as a discretization of the continuum nonlinear Schrödinger equation. The latter is completely integrable and exhibits soliton solutions. However, the application of continuum equations disregarding the inherent discrete lattice structure of the system gives appropriate results only if the spatial extension of the nonlinear wave is much larger than the lattice spacing. On the other hand, dealing with the discrete lattice structures and thus with a network of coupled oscillators one faces the fact that most nonlinear lattice systems are nonintegrable. Only a few examples of exactly solvable discrete lattices are known, such as the Toda lattice [19] and the Ablowitz-Ladik lattice [20] [the case of $\gamma=0$ and $\mu \neq 0$ in Eq. (1)]. Indeed, numerical studies of the dynamics of the standard discrete version of NLS [the case of $\gamma \neq 0$ and $\mu=0$ in Eq. (1)] exhibit nonintegrability (see, e.g., $[21,22]$ ). Hence the standard way of discretizing not only breaks the continuous translational symmetry, but it destroys the integrability at the same time. As a drastic consequence one observes that the exact soliton solution of the integrable NLS becomes pinned under the influence of the periodic lattice potential preventing solitarylike waves from propagating along the lattice chain $[24,25]$. This "soliton trapping" is in sharp contrast to the behavior in the continuum case where the exact (nonstationary) soliton can travel keeping its form invariant. Moreover, in the continuum NLS a soliton of given amplitude can always be moved with any desired velocity through a Galileo boost [26]. On the other hand, the integrable version of the NLS, namely, the Ablowitz-Ladik (AL) equation, has soliton solutions which are the discrete version of the NLS solitons. Due to the complete integrability of the AL system its solitons can travel along the lattice chain without experiencing any pinning potential. For the nonintegrable DNLS the existence of a localized state with frequency lying below the linear phonon band was established $[27,28]$. This solitarylike state reduces to a one-soliton solution in the continuum NLS. However, in DNLS a moving localized state experiences dispersion and eventually decays $[21,27]$. Nevertheless, lack of integrability in the DNLS does not necessarily lead to the absence of (standing) localized states. It is rather so that large-amplitude solitarylike standing excitations in nonintegrable systems appear as well [25].

In order to show the existence of stationary localized excitations in the form of time-periodic and spatially localized solutions for DNLS one can start from the noncoupling limit and show that localized solutions can be maintained for (small) nonzero couplings [29,30]. Recently, MacKay and Aubry have proven the existence of localized solutions in the form of breathers for weakly coupled arrays of oscillators [31]. They also suggested the application of the antiintegrable limit to prove for the DNLS the existence of stationary localized solutions $c_{n}(t)=\phi_{n} \exp (-i \omega t)$ with realvalued time-independent amplitudes $\phi_{n}$ and an oscillation frequency $\omega$ (cf. Sec. 9 of Ref. [31]). Strongly localized states (one-site excitations) are obtained if one lattice oscillator oscillates with a relatively large amplitude while the remaining oscillators perform small-amplitude oscillations decaying exponentially to zero with increasing distance from the excitation peak. Using the noncoupling limit Bressloff proved the existence of localized ground states for the standard diffusive Haken model of a neural network [32]. Since the Haken model is formally equivalent to a Hamiltonian network such as the DNLS one can infer from Bresloff's result that time-periodic and spatially localized solutions ex- 
ist also in the DNLS case. In a recent paper the continuation of localized lattice excitations of zero couplings into stationary localized solutions for nonvanishing couplings for a generalized DNLS (GDNLS) has been given [33]. This GDNLS involves, in addition to nonintegrable DNLS term, the integrable Ablowitz-Ladik contribution and has been introduced recently by Salerno [34] and Cai et al. [28].

In this paper we study the localized stationary solutions of the GDNLS equation in greater detail. We show that the results of the stationary analysis can be used to excite localized stationary states of designed patterns on the lattice. Stationary localized solutions of a pure DNLS system were studied in $[35,36]$ in the context of wave propagation in periodically modulated media. In nonlinear optics Kerr type nonlinearities give rise to DNLS equations and the localized solutions are supported by states in the first gap, therefore called gap solitons [37,38]. The corresponding stationary system can be treated by a nonlinear map approach. In searching for localized solutions one has to be aware that the stationary nonintegrable DNLS system exhibits irregular chaotic behavior which led the authors of $[35,36]$ to the conclusion that perfect localization in a nonintegrable lattice system is impossible. Nevertheless, we demonstrate that stable localized lattice states conspire with the nonanalyticity of the map orbits through homoclinic and heteroclinic connections.

The paper is organized as follows. In Sec. II we describe the generalized AL-DNLS equation. The stationary ALDNLS problem is introduced and linked with a twodimensional area-preserving map. We discuss the stability properties of the fixed points of the map. In Sec. III the Melnikov method is used to prove the existence of homoclinic orbits, thus showing nonanalyticity of the map. With the help of the Birkhoff normal forms we determine homoclinic orbits analytically in Sec. IV and compute the soliton pinning energy. In order to obtain the heteroclinic orbits we exploit a variational approach relating the heteroclinic points to the critical points of a certain action function. In Sec. V we excite bright (dark) solitons in the dynamical DNLS using the homoclinic (heteroclinic) map orbits as initial data. Finally, in Sec. VI we give a short summary.

\section{THE DISCRETE NONLINEAR SCHRÖDINGER EQUATION AND THE STATIONARY PROBLEM}

We study the generalized discrete nonlinear Schrödinger equation

$$
\begin{aligned}
i \frac{d \psi_{n}(t)}{d t}= & -\gamma\left|\psi_{n}(t)\right|^{2} \psi_{n}(t)-\left[V+\mu\left|\psi_{n}(t)\right|^{2}\right] \\
& \times\left[\psi_{n+1}(t)+\psi_{n-1}(t)\right],
\end{aligned}
$$

where $\psi_{n}(t)$ is a complex amplitude, $\gamma$ and $\mu$ are the nonlinearity strengths, and $V$ is the transfer matrix element coupling adjacent oscillators at lattice sites $n$ and $n \pm 1$, respectively. Equation (1) interpolates between the DNLS $(\mu=0)$ and the AL equation $(\gamma=0)$. The AL system is completely integrable and possesses an infinite number of conservation laws for the infinite lattice [20] whereas the DNLS system is nonintegrable $[21,22]$. The combination of the $\mathrm{AL}$ and the DNLS systems, respectively, enables us to treat the DNLS contribution (if $\gamma$ is small) as a nonintegrable perturbation of the integrable AL system (see, e.g., [23]).

We investigate the solution properties of the GDNLS where we focus on time-periodic but spatially localized solutions. Substituting the ansatz

$$
\psi_{n}(t)=\phi_{n} \exp (-i \omega t)
$$

with amplitudes $\phi_{n}$ and the phase (oscillation frequency) $\omega$ into (1), we obtain the following coupled system for the amplitudes $\phi_{n}$ :

$$
\omega \phi_{n}+\gamma\left|\phi_{n}\right|^{2} \phi_{n}+\left(V+\mu\left|\phi_{n}\right|^{2}\right)\left(\phi_{n+1}+\phi_{n-1}\right)=0 .
$$

We are particularly interested in solutions exponentially localized at a single site and distinguish two situations: (1) $\left|\phi_{n}\right|>\left|\phi_{n+1}\right|$ for $n>0$ and $\left|\phi_{n}\right|<\left|\phi_{n+1}\right|$ for $n<0$ with $\lim _{|n| \rightarrow \infty}\left|\phi_{n}\right|=0$ corresponding to the bright solitonlike solution, and (2) $\left|\phi_{n}\right|<\left|\phi_{n+1}\right|$ for $n>0$ and $\left|\phi_{n+1}\right|<\left|\phi_{n}\right|$ for $n<0$ with $\lim _{|n| \rightarrow \infty}\left|\phi_{n}\right|=a>0$ resulting in the dark solitonlike solution. Without loss of generality we assume that both types of the solitonlike solutions have their main deviations from the background around the central element of the lattice. Furthermore we request for the bright (dark) soliton solution exponential decrease (increase) of the amplitudes apart from the central site for $|n| \rightarrow \infty$.

The nonlinear eigenvalue problem to the stationary system of Eq. (1) is cast into an algebraic recursion relation for the amplitudes, viz., $\phi_{n+1}=\phi_{n+1}\left(\phi_{n}, \phi_{n-1}\right)$ which has been studied in $[16,35,36]$ in the limiting cases of $\gamma \neq 0$, $\mu=0$, and of $\gamma=0, \mu \neq 0$ in [39-41], respectively.

It can be readily seen that the current $J$ defined by

$$
J=i\left[\phi_{n}^{*} \phi_{n-1}-\phi_{n} \phi_{n-1}^{*}\right]
$$

is conserved for the system of the stationary equations. Since we consider an open lattice chain (without periodic boundary conditions) we can show that localized solutions imply real amplitudes $\phi_{n} \in \mathbb{R}$ [35]. To this end we consider the value for the current at one of the ends of the chain assumed to be of finite length $N$ for the moment. Representing $\phi_{N-1}$ by the right-hand side of the corresponding stationary equation

$$
V \phi_{N-1}=-\frac{\omega+\gamma\left|\phi_{N}\right|^{2}}{V+\mu\left|\phi_{N}\right|^{2}} \phi_{N},
$$

we immediately obtain that $J \equiv 0$. Due to the conservation of $J$ this result must hold for all lattice indices $n \in[-N, N]$ which, however, can only be fulfilled for either the special case of constant amplitudes $\phi_{n}=$ const or, in general, only for real-valued $\phi_{n} \in R$. Hence for the remainder of this paper we consider real-valued amplitudes $\phi_{n}$.

It is convenient to cast the real-valued second-order difference equation (3) into a two-dimensional map $\mathbb{R}^{2} \rightarrow \mathbb{R}^{2}$ by defining $x_{n}=\phi_{n}$ and $y_{n}=\phi_{n-1}$ where the lattice index plays the role of discrete "time." We arrive at the map

$$
\mathcal{M}:\left\{\begin{array}{l}
x_{n+1}=-\frac{\widetilde{\omega}+\widetilde{\gamma} x_{n}^{2}}{1+\widetilde{\mu} x_{n}^{2}} x_{n}-y_{n} \\
y_{n+1}=x_{n} .
\end{array}\right.
$$


We used the notation $\widetilde{\omega}=\omega / V, \widetilde{\mu}=\mu / V$, and $\tilde{\gamma}=\gamma / V$. For ease of notation we drop the tildes afterwards. Reversibility of the map $\mathcal{M}$ is established by the factorization $\mathcal{M}=\mathcal{M}_{0} \mathcal{M}_{1}$ with

$$
\mathcal{M}_{0}:\left\{\begin{array}{l}
\bar{x}=y \\
\bar{y}=x
\end{array}\right.
$$

and

$$
\mathcal{M}_{1}:\left\{\begin{array}{l}
\bar{x}=x \\
\bar{y}=-\frac{\omega+\gamma x^{2}}{1+\mu x^{2}}-y,
\end{array}\right.
$$

where $\mathcal{M}_{0,1}$ are involutions and their corresponding symmetry lines are given by $S_{0}: x=y$ and $S_{1}: y$ $=-(1 / 2)\left(\omega x+\gamma x^{3}\right) /\left(1+\mu x^{2}\right)$. Furthermore, the map $\mathcal{M}$ is an analytic area-preserving twist map.

In order to investigate stationary localized solutions in the form of the bright (dark) soliton, respectively, it suffices to study the fixed points (period-1 orbits) of the map $\mathcal{M}$. The fixed points, for which $\hat{x}=\hat{y}$, of this map are located at

$$
\hat{x}_{0}=0, \quad \hat{x}_{ \pm}= \pm \sqrt{-\frac{\omega+2}{\gamma+2 \mu}},
$$

where $\hat{x}_{ \pm}$exists only if $\operatorname{sgn}(\omega+2)=-\operatorname{sgn}(\gamma+2 \mu)$. The stability of the fixed points is governed by their value for the corresponding residues [42,43] $\rho=1 / 4\{2-\operatorname{Tr}[D \mathcal{M}(\hat{x})]\}$ and $D$ is the differential operator $(\partial / \partial x, \partial / \partial y)$. The tangent map $D \mathcal{M}$ is determined by

$$
\operatorname{DM}(x)=\left(\begin{array}{cc}
\omega_{n} & -1 \\
1 & 0
\end{array}\right),
$$

with

$$
\omega_{n}=-\frac{\omega+(3 \gamma-\mu \omega) x_{n}^{2}+\gamma \mu x_{n}^{4}}{\left(1+\mu x_{n}^{2}\right)^{2}} .
$$

The residue corresponding to the fixed point at the origin is

$$
\rho=\frac{1}{4}(\omega+2) .
$$

For $\omega$ values within the range of the linear band, i.e., $|\omega|<2,0<\rho<1$ holds and the origin is a stable elliptic fixed point encircled by stable elliptic type map orbits. For $|\omega|>2$ (outside the range of the linear band) we distinguish the following two cases.

(i) $\omega<-2, \gamma+2 \mu>0$ : In this case the residue passes through zero, i.e., $\rho<0$, and hence the origin loses stability and is turned into an unstable hyperbolic point caused by a tangent bifurcation. This hyperbolic point is connected to itself by a homoclinic orbit created by the (invariant) unstable and stable manifold. As will be shown below, the homoclinic orbit is manifested on the lattice chain as a soli- tonlike solution which is equivalent to the so-called gap soliton of nonlinear optics lying in the stop band below the linear passing band $[37,44]$.

The pair of points $\hat{x}_{ \pm}$on the symmetry line $S_{0}$ form stable elliptic fixed points.

(ii) $\omega>2, \gamma+2 \mu<0$ : The value for the residue at the origin passes through the value of one, that is, $\rho>1$, connected with the onset of a period-doubling bifurcation, where the fixed point is converted into an unstable hyperbolic point with reflection. The newly created period- 2 orbits are located on the line $x=-y$.

The homoclinic map orbit supports on the lattice chain a solitonlike solution which exists in the gap above the linear passing band and has alternating signs for adjacent amplitudes, i.e., $\operatorname{sgn}\left(\phi_{n+1}\right)=-\operatorname{sgn}\left(\phi_{n}\right)$ as a characteristic feature (see below). This stationary localized structure has been called a staggered soliton by Cai, Bishop, and GronbechJensen in their study of the combined AL-DNLS equation [28]. Correspondingly the soliton solution of case (i) is called the unstaggered soliton. Note that upon sign change $\gamma \rightarrow-\gamma$ and $\omega \rightarrow-\omega$ the map has the symmetry property of $\operatorname{sgn}\left(\phi_{n+1}\right)=-\operatorname{sgn}\left(\phi_{n}\right)$ so that the unstaggered and staggered soliton replace each other.

A third case of unstable fixed points can also be attributed to the occurrence of a stationary localized structure on the nonlinear lattice, as follows.

(iii) $|\omega|<2$ and $\gamma+2 \mu<0$ : Since the frequency is in the linear band the origin is still a stable elliptic fixed point whereas the pair of fixed points $\hat{x}_{ \pm}$on the symmetry line $S_{0}$ represents two unstable hyperbolic fixed points which are connected to each other via a (pair of) heteroclinic orbits. This heteroclinic map orbit represents a kinklike solution, also called a dark soliton. There exist staggered and unstaggered versions of this soliton, too.

Since the nature of the solitonlike solutions depends on the stability of the fixed points we summarize their bifurcation behavior. The stability properties of the fixed point $\left(\hat{x}_{0}, \hat{y}_{0}\right)$ are

$$
|\omega|<2 \text {, elliptic point, }
$$

$\omega>2$, hyperbolic point with reflection,

$$
\omega<-2 \text {, hyperbolic point. }
$$

The bifurcation behavior of the fixed points $\left(\hat{x}_{ \pm}, \hat{y}_{ \pm}\right)$is appropriately illustrated in the $\gamma$ versus $\omega$ parameter plane for fixed values of $\mu$ (Fig. 1). Different lines are drawn to separate the parameter regions in which the residue takes on the values of zero and one, respectively. The residue is given by

$$
\rho=-\frac{1}{2} \frac{(\omega+2)(\gamma+2 \mu)}{\gamma-\mu \omega} .
$$

Furthermore the parameter ranges for the existence of the different soliton types are indicated on the plot. Comparing Figs. 1(a) and 1(b), belonging to $\mu=1$ and $\mu=2$, respectively, we note that increasing the integrability parameter $\mu$ shifts the $\rho=1$ lines on the $\gamma-\omega$ plane to higher $|\gamma|$ values. This means that higher $\gamma$ and $\omega$ values are needed to excite a staggered bright soliton when $\mu$ is enhanced. On the other hand, increasing $\mu$ demands higher $|\gamma|$ to get below the $\rho<0$ line where dark solitons exist. We remark that the dark soliton solution exists only for $\gamma<0$ and not in the case of 

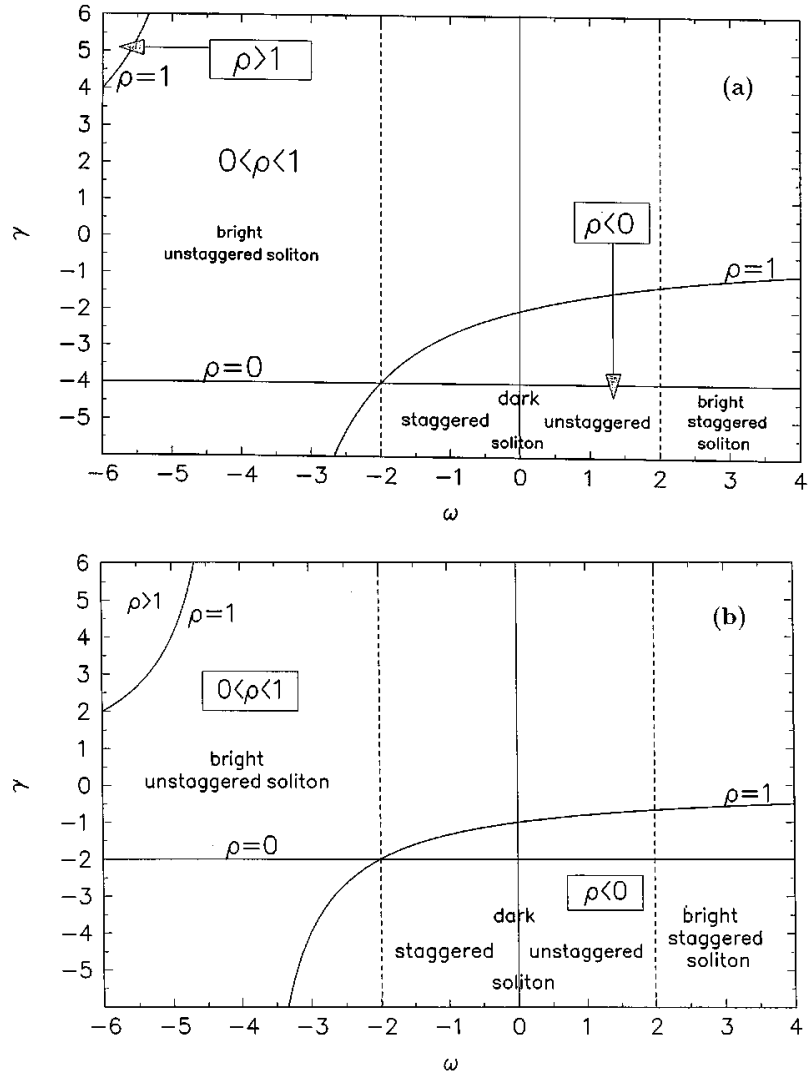

FIG. 1. Bifurcation behavior illustrated in the $\gamma$ versus $\omega$ parameter plane. The regions of different values of the residue $\rho$ are marked. (a) Parameter $\mu=1$. (b) Parameter $\mu=2$.

vanishing $\gamma$. In other words, AL dark solitons are excluded whereas the bright solitons exist in both extreme cases of $(\gamma \neq 0, \mu=0)$ and $(\gamma=0, \mu \neq 0)$.

In Fig. 2(a) we show the staggered and unstaggered bright solitons superimposed on a single map plane for the parameter values of $\omega=2.25, \mu=1, \gamma=-5$ and $\omega=-2.25$, $\mu=1, \gamma=3$, respectively. Not visible on this scale are the transversal intersections of the stable and unstable manifolds of the hyperbolic point at the origin. We superimposed the symmetry lines $S_{0}$ and $S_{1}$. Of special importance is that for area-preserving reversible maps a class of homoclinic orbits has one of its points on the dominant symmetry line [45] which, in our case, is $S_{1}$.

The next section is devoted to proving analytically the existence of these intersections. Figure 2(b) illustrates the heteroclinic structure for the parameters $\omega=-1, \gamma=-3$, $\mu=1$. The chaotic layer connected with the heteroclinic connection of the two hyperbolic points at $\left(\hat{x}_{ \pm}, \hat{y}_{ \pm}\right)$is clearly seen.

\section{THE MELNIKOV FUNCTION AND HOMOCLINIC ORBITS}

As is well known in generic nonintegrable maps the stable and unstable manifolds of hyperbolic equilibria cross each other in homoclinic points; or there might appear crossings
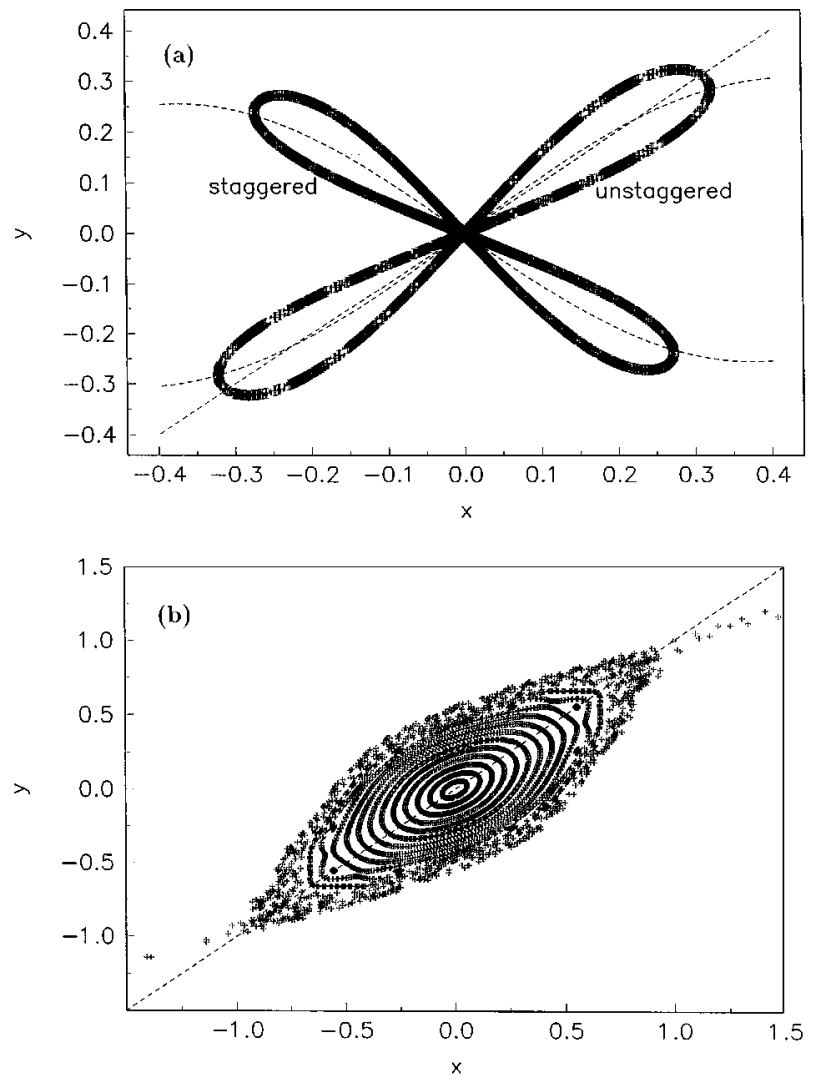

FIG. 2. Map orbits related to the hyperbolic points at $\left(\hat{x}_{0}, \hat{y}_{0}\right)$ and $\left(\hat{x}_{ \pm}, \hat{y}_{ \pm}\right)$. (a) The homoclinic orbits related to the staggered and unstaggered bright solitons, respectively. Parameters: $\gamma=5, \mu=1$, and $\omega=2.25$. (b) The heteroclinic orbit related to the unstaggered dark soliton for the parameters $\gamma=3, \mu=1$, and $\omega=-2.25$. Also shown are some orbits in the interior of the heteroclinic connection.

of the stable and the unstable manifolds of different hyperbolic points, called heteroclinic connections. Such twodimensional maps are often associated with the Poincaré map of periodically perturbed two-dimensional flows [42]. For these time-continuous flows the Melnikov function proved to be a powerful method to show the existence of orbits homoclinic to a hyperbolic equilibrium. Glasser et al. [46] extended the Melnikov analysis to two-dimensional discrete maps of the plane which are of the form $u_{n+1}$ $=F\left(u_{n}\right)+\epsilon G\left(u_{n}\right)$ with $u=(x, y) \in \mathcal{R}^{2}$. Thus the right-hand side is assumed to consist of a completely integrable part $F$ and a small nonintegrable perturbation $\epsilon G$ with $\epsilon \ll 1$. Furthermore, the unperturbed system of $\epsilon=0$ possesses an unstable equilibrium characterized by coinciding stable and unstable manifolds forming a perfect unperturbed separatrix on which the solution is known explicitly. Based on geometric arguments a Melnikov function was developed in [46] measuring the distance between the stable and unstable manifolds under the action of the perturbation.

For a perturbational treatment we consider the nonlinear term related with $\gamma$ as a small (nonintegrable) perturbation of the integrable $\mathrm{AL}$ map $(\gamma=0, \mu \neq 0)$. Therefore we introduce in (6) the small parameter $\epsilon$ : 


$$
\begin{gathered}
x_{n+1}=-\frac{\omega+\epsilon \gamma x_{n}^{2}}{1+\mu x_{n}^{2}} x_{u}-y_{n}, \\
y_{n+1}=x_{n},
\end{gathered}
$$

whose integrable part ( $\epsilon=0)$ possesses a separatrix given by

$$
\mu x^{2} y^{2}+x^{2}+y^{2}+\omega x y=0 .
$$

In the first quadrant the separatrix loop can be parametrized by

$$
\begin{gathered}
x_{n}(t)=\frac{1}{\sqrt{\mu}} \sinh \beta \operatorname{sech}(t-n \beta), \\
y_{n}(t)=\frac{1}{\sqrt{\mu}} \sinh \beta \operatorname{sech}[t-(n+1) \beta],
\end{gathered}
$$

where $\cosh \beta=-\omega / 2$ and $t$ is a real parameter regulating the position on the separatrix loop. Note that the AL soliton center of $x_{0}(0)=\sqrt{\left(\omega^{2} / 4-1\right) / \mu}$ and $y_{0}(0)=-2 x_{0}(0) / \omega$ is determined on the map plane by the intersection point of the AL separatrix loop with the symmetry line $S_{1}$.

According to [46] the Melnikov function is given by

$$
M(t ; \omega, \mu, \gamma)=\left\|u_{0}(t)\right\| \Delta^{\prime}(0),
$$

with

$$
\Delta^{\prime}(0)=\sum_{k=-\infty}^{\infty} G\left(x_{k-1}, y_{k-1}\right) \wedge v_{k},
$$

where the wedge product is $\left(u_{1}, u_{2}\right) \wedge\left(v_{1}, v_{2}\right)=u_{1} v_{2}$ $-u_{2} v_{1}$. Therefore the unit tangent vector to the separatrix is given as

$$
v_{k}(t)=u_{k}(t) /\left\|u_{0}(t)\right\|
$$

where

$$
u_{k}(t)=\left(-y_{k}-\frac{\omega}{2} x_{k}-\mu x_{k}^{2} y_{k}, x_{k}+\frac{\omega}{2} y_{k}+\mu x_{k} y_{k}^{2}\right) .
$$

In our case the perturbation is $G\left(x_{n}\right)=\left(-\epsilon \gamma x_{n}^{3} /\right.$ $\left.\left(1+\mu x_{n}^{2}\right), 0\right)$. Thus we have

$$
\begin{aligned}
\Delta^{\prime}(0) & =-\epsilon \gamma \sum_{k=-\infty}^{\infty} \frac{x_{k-1}^{3}}{1+\mu x_{k-1}^{2}}\left(x_{k}+\frac{\omega}{2} x_{k-1}+\mu x_{k} x_{k-1}^{2}\right) \\
& =\frac{\epsilon \gamma}{\omega} \sum_{k=-\infty}^{\infty}\left(x_{k+1}+x_{k-1}\right) x_{k}^{2}\left(x_{k+1}+\frac{\omega}{2} x_{k}+\mu x_{k+1} x_{k}^{2}\right) \\
& =\frac{\epsilon \gamma}{\omega} \sum_{k=-\infty}^{\infty}\left(x_{k}^{2} x_{k+1}^{2}+\omega x_{k}^{3} x_{k+1}+\mu x_{k}^{4} x_{k+1}^{2}+x_{k+1}^{4}\right) .
\end{aligned}
$$

We therefore need to calculate sums of the form $S_{1}(a, b, \lambda)=\sum_{k=-\infty}^{\infty} \operatorname{sech}^{2}(\lambda n-a) \operatorname{sech}^{2}(\lambda n-b)$. Using the Poisson summation formula we have

$$
\begin{aligned}
S_{1}(a, b, \lambda)= & \sum_{k=-\infty}^{\infty} \int_{-\infty}^{\infty} \exp (2 \pi i n x) \operatorname{sech}^{2}(\lambda x-a) \\
& \times \operatorname{sech}^{2}(\lambda x-b) d x .
\end{aligned}
$$

The integral is evaluated using residue calculus:

$$
\begin{gathered}
\int_{-\infty}^{\infty} \exp (-\alpha x) \operatorname{sech}^{2}(\lambda x-a) \operatorname{sech}^{2}(\lambda x-b) d x \\
=-\frac{\pi}{\lambda} \operatorname{cosec}\left(\frac{\alpha \pi}{2 \lambda}\right) \operatorname{cosech}^{2} \delta\left\{\frac { \alpha } { \lambda } \left(\exp \left[-\frac{\alpha a}{\lambda}\right]\right.\right. \\
\left.+\exp \left[-\frac{\alpha b}{\lambda}\right]\right)+2 \operatorname{coth} \delta\left(\exp \left[-\frac{\alpha a}{\lambda}\right]\right. \\
\left.\left.\quad-\exp \left[-\frac{\alpha b}{\lambda}\right]\right)\right\},
\end{gathered}
$$

where $\delta=a-b$. Setting $\alpha=-2 \pi i n$ and performing the remaining sum we get

$$
S_{1}(a, b, \lambda)=\frac{2 \pi}{\lambda}[h(\delta) \bar{I}(a, b, \lambda)+f(\delta) I(a, b, \lambda)],
$$

with

$$
\begin{gathered}
I=\phi\left(\frac{\pi b}{\lambda}, \frac{\pi^{2}}{\lambda}\right)-\phi\left(\frac{\pi a}{\lambda}, \frac{\pi^{2}}{\lambda}\right)+\frac{\delta}{\pi}, \\
\bar{I}=-\frac{\partial \phi}{\partial b}-\frac{\partial \phi}{\partial a}-\frac{2}{\pi},
\end{gathered}
$$

where

$$
\begin{aligned}
\phi(x, u) & =\sum_{n=1}^{\infty} \sin (2 n u) \operatorname{cosech}(n x) \\
& =\frac{K}{\pi}\{E[a m(2 K u / \pi)]-2 u E / \pi\}
\end{aligned}
$$

and finally $h(\delta)=\operatorname{cosech}^{2} \delta$ and $f(\delta)=2 \operatorname{cosech} \delta \operatorname{coth} \delta$. Similarly, we have

$$
\begin{aligned}
S(a, b, \lambda) & =\sum_{n=-\infty}^{\infty} \operatorname{sech}(\lambda n-a) \operatorname{sech}(\lambda n-b) \\
& =g(\delta) I(a, b, \lambda),
\end{aligned}
$$

where $g(\delta)=\operatorname{cosech} \delta$. The sum $S_{2}(a, b, \lambda)$ $=\sum_{n=-\infty}^{\infty} \operatorname{sech}^{3}(\lambda n-a) \operatorname{sech}(\lambda n-b)$ follows from $S_{2}$ $=\left(S-S^{\prime \prime}\right) / 2$, where the primes denote derivatives with respect to $a$. The sum $S_{3}(a, b, \lambda)=\sum_{n=-\infty}^{\infty} \operatorname{sech}^{4}(\lambda n$ $-a) \operatorname{sech}^{2}(\lambda n-b)$ can be constructed as $S_{3}=\frac{2}{3} S_{1}-\frac{1}{6} S_{1}^{\prime \prime}$ while the last sum $\sum_{n=-\infty}^{\infty} \operatorname{sech}^{4}(\lambda n-a)$ is $S_{2}(a, a, \lambda)$. Thus we obtain

$$
\begin{aligned}
\Delta^{\prime}(0)= & \frac{\epsilon \gamma}{\omega \mu} \sinh ^{4} \beta\left[S_{1}(t, t-\beta, \beta)-2 \cosh \beta S_{2}(t, t-\beta, \beta)\right. \\
& \left.+\sinh ^{2} \beta S_{3}(t, t-\beta, \beta)+S_{2}(t, t, \beta)\right]
\end{aligned}
$$


which reduces to

$$
\begin{aligned}
\Delta^{\prime}(0)= & -\frac{\epsilon \gamma}{\omega \mu} \sinh ^{4} \beta\left\{4 \operatorname{coth} \beta\left(\frac{K^{3} k^{2}}{\beta^{2}}\right) \operatorname{dn}\left[\frac{2 K t}{\beta}\right] \operatorname{sn}\left[\frac{2 K t}{\beta}\right]\right. \\
& \times \operatorname{cn}\left[\frac{2 K t}{\beta}\right]+\frac{8}{3} \frac{K^{4} k^{2}}{\beta^{3}}\left(\operatorname{dn}^{2}\left[\frac{2 K t}{\beta}\right] \operatorname{cn}^{2}\left[\frac{2 K t}{\beta}\right]\right. \\
& \left.\left.-\operatorname{dn}^{2}\left[\frac{2 K t}{\beta}\right] \operatorname{sn}^{2}\left[\frac{2 K t}{\beta}\right]-k^{2} \operatorname{sn}^{2}\left[\frac{2 K t}{\beta}\right] \operatorname{cn}^{2}\left[\frac{2 K t}{\beta}\right]\right)\right\},
\end{aligned}
$$

where $E(k) / K(k)=\pi / \beta$, and $K$ and $E$ are the complete elliptic integral of the second kind and the associated complete elliptic integral of the second kind, respectively, and dn, sn, and $\mathrm{cn}$ are Jacobian elliptic functions. Equation (32) shows that the Melnikov function is a periodic function of $t$ with an infinite number of simple zeros proving the presence of homoclinic chaos in the perturbed map. As a result of the nonintegrability the stable and unstable manifolds of the hyperbolic fixed points of the perturbed map are no longer identical, but intersect and create a homoclinic tangle. Equation (32) also shows that the separatrix splitting is proportional to the ratio $\gamma /(\omega \mu)$. The same ratio was found in [33] to limit the parameter region where the map (6) shows regular motion. Finally, from Eq. (32) it is obtained that the distance between successive transversal intersections of the stable and unstable manifolds purely depends on the ratio $K / \beta$, which in turn only depends on $\omega$ and not directly on the nonlinearity parameters $\gamma$ and $\mu$. The nonlinearity parameters appear only in front of the Melnikov function (33) as a factor regulating the degree of the separatrix splitting. Apparently with higher nonintegrability parameter $\gamma$ the splitting grows whereas the integrability parameter $\mu$ acts in the opposite direction, namely, suppresses the splitting.

According to the defining relation between $k$ and $\omega$ we can obtain that $k$ has a rather slow dependence on $\omega$ which means that $k$ can be considered small even for rather large $\omega$ such that it is reasonable to consider $k$ as small in Eq. (32). This approximation reduces the complexity of $\Delta^{\prime}(0)$ considerably. Using small $k$ expansions of the Jacobian elliptic functions [47]

$$
\Delta^{\prime}(0)=-\frac{\epsilon \gamma}{\mu} A(\omega) \cos \left(\frac{4 K}{\beta} t+\theta\right)+O\left(k^{2}\right),
$$

where

$$
\begin{gathered}
A(\omega)=\frac{2 K^{3} k^{2}}{\omega \beta^{2}} \sqrt{\operatorname{coth}^{2} \beta+\frac{26 K^{2}}{9 \beta^{2}}} \sinh ^{4} \beta, \\
\tan \theta=\frac{3 \beta \operatorname{coth} \beta}{4 K} .
\end{gathered}
$$

The distance between successive zeros of the Melnikov function is given by

$$
\Delta t=\frac{\pi \beta}{4 K} \simeq \frac{1}{2} \ln \left(-\frac{\omega}{2}+\sqrt{\frac{\omega^{2}}{4}-1}\right) \equiv \frac{1}{2} \ln (\lambda),
$$

telling us that the distance between two adjacent intersections of the stable and unstable manifold depends solely on the oscillation frequency $\omega$. The distance vanishes at the band edge of $\omega=-2$ and grows logarithmically when $\omega$ ranges further down in the gap. Interestingly, the quantity $\lambda$ in Eq. (35) is identical to the maximal eigenvalue of the linearized map around the hyperbolic point at the origin.

\section{NORMAL FORM COMPUTATION OF THE HOMOCLINIC ORBIT}

In this section we use the Birkhoff normal forms to compute the homoclinic orbit corresponding to the unstable hyperbolic point at the map origin for $\omega<-2$ and $\gamma+2 \mu>0$. The Birkhoff normal form of an area-preserving map yields a simplified version of the map achieved by a canonical transformation in the form of a formal series expansion [48]. Normal forms are powerful tools for analytical determination of homoclinic orbits of two-dimensional maps. Recently Tabacman [49] developed another method for computing homoclinic and heteroclinic orbits relying on an action principle. In a subsequent section we exploit this method to obtain the orbit heteroclinic to the fixed points $\left(\hat{x}_{ \pm}, \hat{y}_{ \pm}\right)$. Later we need the "exact" location of the intersection points of the stable and unstable manifolds to use them as initial data in order to excite stationary localized states of the GDNLS.

We begin by rewriting the map of (6) as follows:

$$
\mathcal{M}:\left\{\begin{array}{l}
\bar{x}=-\omega x-\omega \frac{(\gamma / \omega-\mu) x^{2}}{1+\mu x^{2}} x-y \\
\bar{y}=x .
\end{array}\right.
$$

The linear part

$$
\begin{gathered}
\bar{x}=-\omega x-y, \\
\bar{y}=x .
\end{gathered}
$$

is diagonalized through the canonical transformation

$$
P=\lambda^{+} x-y, \quad Q=\lambda^{-} x-y,
$$

and

$$
\lambda^{ \pm}=\frac{1}{2}\left[-\omega \pm \sqrt{\omega^{2}-4}\right]
$$

are the eigenvalues of the linear transformation (37). The inverse transformation is given by

$$
x=\frac{P-Q}{\lambda^{+}-\lambda^{-}}, \quad y=\frac{\lambda^{-} P-\lambda^{+} Q}{\lambda^{+}-\lambda^{-}} .
$$

After a scaling $P \rightarrow \sqrt{\mu} P$ and $Q \rightarrow \sqrt{\mu} Q$ and with the help of $\lambda \equiv \lambda^{+}=1 / \lambda^{-}$we obtain for the transformed map

$$
\begin{aligned}
\bar{P}= & \lambda P-\left(\frac{\gamma}{\mu}-\omega\right) \frac{\lambda^{4}}{\left(\lambda^{2}-1\right)^{3}}(P-Q)^{3} \\
& \times \frac{1}{1+\left[1 /(\lambda-1 / \lambda)^{2}\right](P-Q)^{2}},
\end{aligned}
$$


and

$$
\begin{aligned}
\bar{Q}= & \frac{1}{\lambda} Q-\left(\frac{\gamma}{\mu}-\omega\right) \frac{\lambda^{2}}{\left(\lambda^{2}-1\right)^{3}}(P-Q)^{3} \\
& \times \frac{1}{1+\left[1 /(\lambda-1 / \lambda)^{2}\right](P-Q)^{2}},
\end{aligned}
$$

or its equivalent Taylor expansion about the origin,

$$
\begin{aligned}
& \bar{P}=\lambda P+\left(\frac{\gamma}{\mu}-\omega\right) \lambda \sum_{n=1}^{\infty}(-1)^{n}\left[\frac{\lambda}{\left(\lambda^{2}-1\right)}(P-Q)\right]^{(2 n+1)} \\
& \bar{Q}=\frac{1}{\lambda} Q+\left(\frac{\gamma}{\mu}-\omega\right) \frac{1}{\lambda} \sum_{n=1}^{\infty}(-1)^{n}\left[\frac{\lambda}{\left(\lambda^{2}-1\right)}(P-Q)\right]^{(2 n+1)}
\end{aligned}
$$

Birkhoff [48] introduced a canonical transformation based on the series expansion

$$
\begin{aligned}
& P=\xi+\sum_{k=2}^{\infty} \sum_{l=0}^{k} p_{k l} \xi^{k-l} \eta^{l}, \\
& Q=\eta+\sum_{k=2}^{\infty} \sum_{l=0}^{k} q_{k l} \xi^{k-l} \eta^{l},
\end{aligned}
$$

such that the $(\xi, \eta)$ map is given by

$$
\begin{gathered}
\bar{\xi}=U(\xi \eta) \xi, \\
\bar{\eta}=[U(\xi \eta)]^{-1} \eta .
\end{gathered}
$$

The function $U$ depends only on the product $\xi \eta$ and has a formal series expansion

$$
U(\xi \eta)=\lambda\left(1+\sum_{k=1}^{\infty} U_{2 k}(\xi \eta)^{k}\right) .
$$

Moser [50] proved the convergence of the series (47)-(49) in a disk surrounding the origin provided the series in (43) and (44) represent analytical functions, which is true in our case. Moreover, it was shown that whenever the inverse map is also analytic, the region of convergence of the series can be extended in narrow strips along the stable and unstable manifolds, respectively [51]. Furuya and Ozorio de Almeida [52] used the Birkhoff normal form for a precise computation of homoclinic points of the standard map and our approach proceeds along the same lines for the AL-DNLS map.

It is useful to define the auxiliary series

$$
(P-Q)^{2 n+1}=\sum_{k=2 n+1}\left(d^{2 n+1}\right)_{k l} \xi^{k-l} \eta^{l}
$$

The recursion relations for the expansion coefficients are then determined by

$$
\begin{aligned}
& \lambda U_{k-1} \delta_{k, 2 l+1}-\lambda p_{k l} \\
& =\left(\frac{\gamma}{\mu}-\omega\right) \lambda \sum_{n=1}^{(k-1) / 2}(-1)^{n}\left(d^{2 n+1}\right)_{k l}\left(\frac{\lambda}{\lambda^{2}-1}\right)^{2 n+1} \\
& \quad-\lambda^{k-2 l} \sum_{n=0} p_{k-2 n, l-n}\left(U^{k-2 l}\right)_{2 n}
\end{aligned}
$$

$$
\begin{aligned}
& \lambda\left(U^{-1}\right)_{k-1} \delta_{k, 2 l-1}-\frac{1}{\lambda} q_{k l} \\
& =\left(\frac{\gamma}{\mu}-\omega\right) \frac{1}{\lambda} \sum_{n=1}^{(k-1) / 2}(-1)^{n}\left(d^{2 n+1}\right)_{k l}\left(\frac{\lambda}{\lambda^{2}-1}\right)^{2 n+1} \\
& \quad-\lambda^{k-2 l} \sum_{n=0} q_{k-2 n, l-n}\left(U^{k-2 l}\right)_{2 n}
\end{aligned}
$$

The stable and unstable manifold of the map $\mathcal{M}$ are the images of the $\eta=0$ and the $\xi=0$ axes under the transformation $U$. Since the Melnikov function possesses infinitely many simple zeros the stable and unstable manifold cross each other in homoclinic points which we can compute from the images of the two axes under $U$. This method provides the homoclinic orbit with uniform precision. The unstable manifold as the projection on the $\xi$ axis is determined by the odd-power series

$$
P=\xi+\sum_{k=3}^{\infty} p_{k 0} \xi^{k}, \quad Q=\sum_{k=3}^{\infty} q_{k 0} \xi^{k}
$$

for which the coefficients $p_{k 0}$ and $q_{k 0}$ can be given in closed form

$$
\begin{gathered}
p_{k 0}=\left(\frac{\gamma}{\mu}-\omega\right) \lambda \frac{1}{\lambda^{k}-\lambda} \sum_{n=1}^{(k-1) / 2}(-1)^{n}\left(\frac{\lambda}{\lambda^{2}-1}\right)^{2 n+1}\left(d^{2 n+1}\right)_{k 0} \\
\simeq\left(\frac{\gamma}{\mu}-\omega\right) \lambda \frac{1}{\lambda^{k}-\lambda}(-1)^{(k-1) / 2}\left(\frac{\lambda^{2}}{\lambda^{2}-1}\right)^{k} \\
q_{k 0}=\left(\frac{\gamma}{\mu}-\omega\right) \frac{1}{\lambda} \frac{1}{\lambda^{k}-1 / \lambda} \\
\quad \times \sum_{n=1}^{(k-1) / 2}(-1)^{n}\left(\frac{\lambda}{\lambda^{2}-1}\right)^{2 n+1}\left(d^{2 n+1}\right)_{k 0} \\
\simeq\left(\frac{\gamma}{\mu}-\omega\right) \frac{1}{\lambda} \frac{1}{\lambda^{k}-1 / \lambda}(-1)^{(k-1) / 2}\left(\frac{\lambda^{2}}{\lambda^{2}-1}\right)^{k}
\end{gathered}
$$

We have omitted terms of order higher than $\lambda^{-2}$. Inserting (54) and (55) into (53) we obtain 

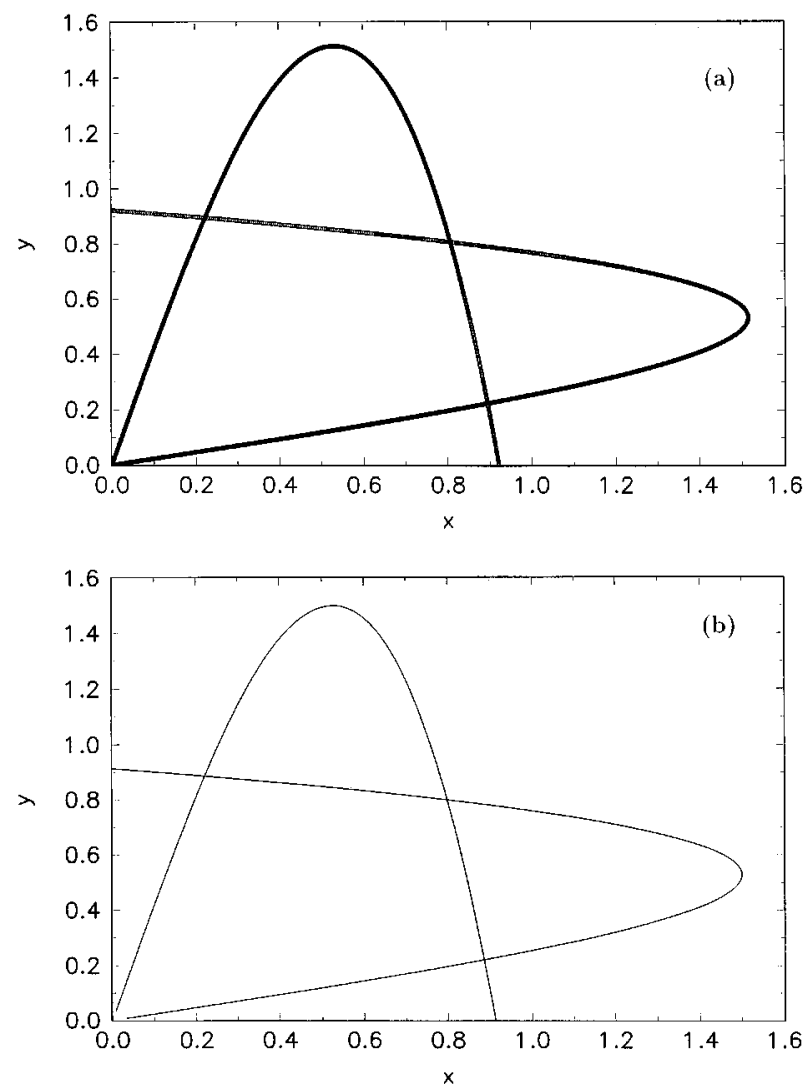

FIG. 3. First homoclinic windings for the parameters: $\gamma=1$, $\mu=0$, and $\omega=-0.8$ and $V=0.2$. (a) The orbit obtained from the map (6). (b) Result of the normal form expressions (53).

$P=\xi+\left(\frac{\gamma}{\mu}-\omega\right) \lambda \sum_{k=3}^{\infty} \frac{1}{\lambda^{k}-\lambda}(-1)^{(k-1) / 2}\left(\frac{\lambda^{2}}{\lambda^{2}-1}\right)^{k} \xi^{k}$.

If again terms of the order higher than $\lambda^{-2}$ are dropped the series can be summed up yielding

$$
P=\xi-\left(\frac{\gamma}{\mu}-\omega\right) \frac{\xi}{1+(\xi / \lambda)^{2}}\left(\frac{\xi}{\lambda}\right)^{2} .
$$

Correspondingly, we obtain

$$
\begin{aligned}
Q & =\left(\frac{\gamma}{\mu}-\omega\right) \frac{1}{\lambda} \sum_{k=3}^{\infty} \frac{1}{\lambda^{k}-1 / \lambda}(-1)^{(k-1) / 2}\left(\frac{\lambda^{2}}{\lambda^{2}-1}\right)^{k} \xi^{k} \\
& =-\left(\frac{\gamma}{\mu}-\omega\right) \frac{\xi^{3}}{\lambda^{4} 1+(\xi / \lambda)^{2}}+O\left(\lambda^{-2}\right) .
\end{aligned}
$$

Using the inverse transformation of Eq. (40) the unstable manifold is determined by

$$
x=\lambda y\left\{1+(\gamma-\omega \mu)\left(1-\frac{1}{\lambda^{2}}\right)^{3} \frac{y^{2}}{\lambda^{2}+\left(\lambda^{2}-1\right)^{2} \mu y}\right\} .
$$

Apparently there is no intersection for $\gamma=\omega \mu$ for which the map degenerates to a linear one. Since the map orbits obey the symmetry $x \leftrightarrow y$ the stable manifold is obtained from Eq. (60) by exchanging $x$ and $y$.

In Fig. 3(a) we show the first intersections of stable and unstable manifolds obtained from the map $\mathcal{M}$. Figure 3(b) depicts the manifolds derived with the help of the normal form expression (60). A comparison of the two results reveals the high accuracy of the normal form computations.

\section{HOMOCLINIC, HETEROCLINIC ORBITS, AND EXCITATIONS OF LOCALIZED SOLUTIONS}

We have seen in Sec. II that in the map plane the origin $\left(x_{n}, y_{n}\right) \equiv\left(\phi_{n+1}, \phi_{n}\right)=(0,0)$ forms a hyperbolic fixed point $p$ as long as $|\omega|>2$ which possesses its invariant stable and unstable manifolds. Points belonging to the stable manifold $\mathcal{W}^{s}(p)$ approach the fixed point $p$ under map iteration $\mathcal{M}^{n}$ for $n \rightarrow \infty$, likewise points on the unstable manifold $\mathcal{W}^{u}(p)$ reach the fixed point $p$ for $n \rightarrow-\infty$. Thus going along the invariant manifolds of the hyperbolic fixed point localized stationary solutions could be created. However, searching for solitonlike solutions, one has to be aware that the DNLS system is nonintegrable; a fact which normally prevents it from having solitonlike solutions, since these are associated with an integrable system. As already mentioned, the integrable Ablowitz-Ladik equation possesses soliton solutions which are the discrete versions of the solitons of the (integrable) continuum nonlinear Schrödinger equation [20]. These discrete AL solitons manifest in the integrable map as a perfect separatrix with coinciding stable and unstable manifold. Since the DNLS system is nonintegrable (see Sec. III) we know that the separatrix is destroyed in the sense that the stable and unstable manifolds no longer coincide but rather intersect each other transversally in homoclinic points, creating complicated chaotic dynamics developing eventually Smale horseshoes. The relation between homoclinic and heteroclinic orbits of nonintegrable maps with localized solutions of the underlying lattice system generating the map is known since the pioneering work of Aubry and co-workers [53,54]. Aubry and Le Daeron [54] studied the FrenkelKontorova model consisting of an infinite sequence of equal springs and masses under the action of a periodic potential. They interpreted the Frenkel-Kontorova model as a generating variational for the orbits of the standard map and showed that homoclinic (heteroclinic) intersections, called also discommensurations, are attributed to localized states pinned by the lattice. (We refer to the next section for details.) Coste and Peyrard [35] as well as Wan and Soukoulis [36] dealt with the linkage between the homoclinic orbit of the DNLS map and localized states of the lattice. Coste and Peyrard draw the conclusion that "perfect localization in a DNLS system is impossible" because of the residual stochasticity near the hyperbolic points. Instead of exhibiting a " one-peak solution" as in an integrable system where a solution can approach a hyperbolic point as arbitrarily close as is wanted, they claim that in a nonintegrable system multipeak solutions are expected to appear. Wan and Soukoulis came to the same conclusion regarding the DNLS system in the context of Holstein's polaron model. They interpreted the homoclinic chaos with its stochastic behavior of the map orbits in the vicinity of the hyperbolic point as a splitting of the large polaron solution (represented by a solitonlike orbit) into an array of randomly distributed small polarons pinned by the discrete lattice [36].

In contrast to the propositions in $[35,36]$, there exist stable 


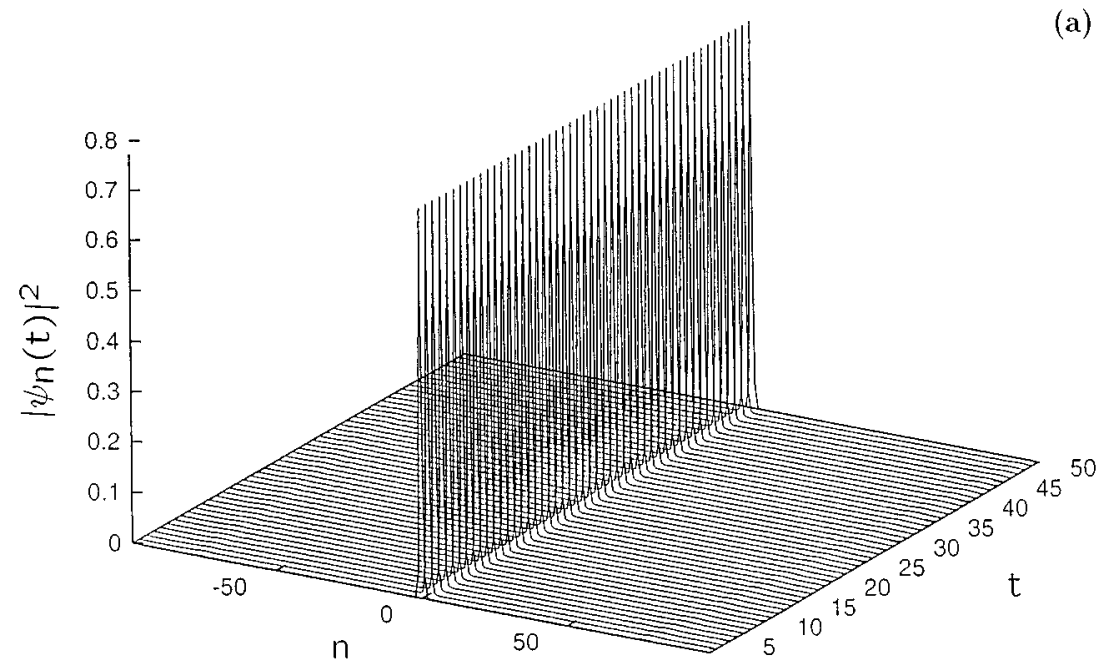

(a)

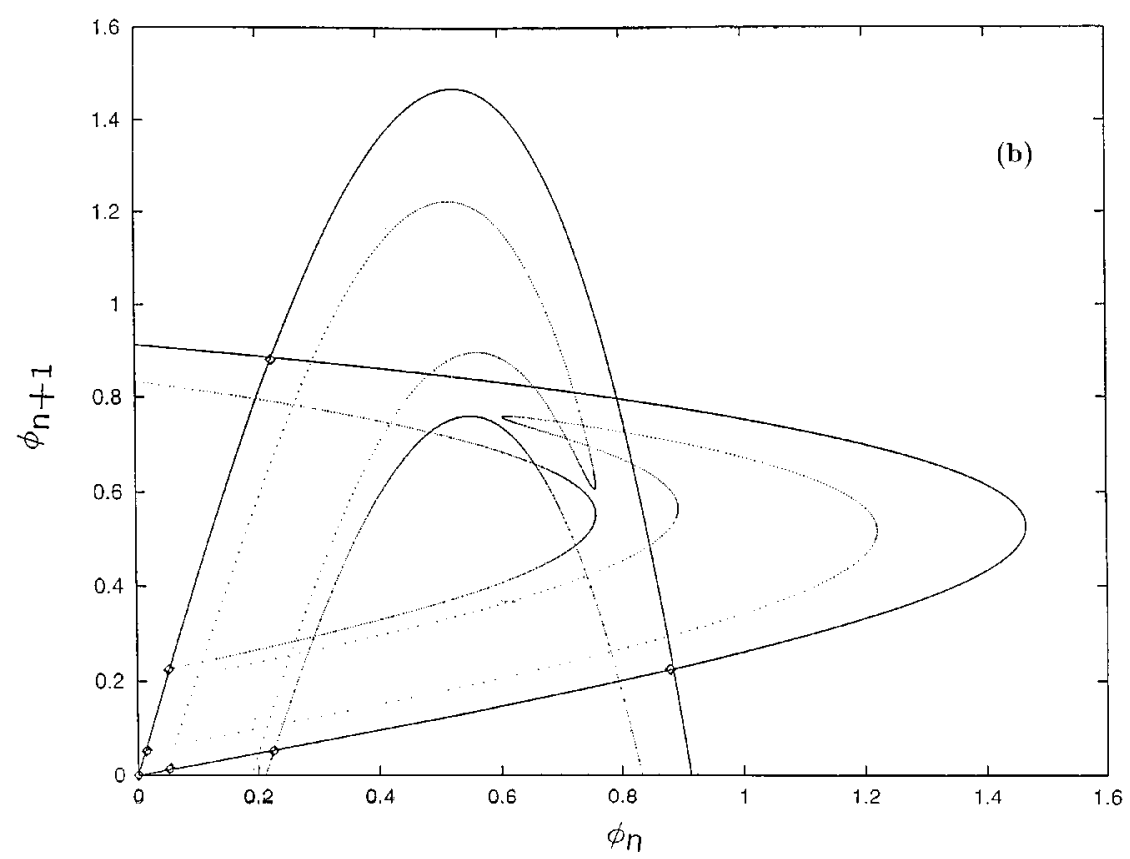

FIG. 4. (a) Profile $\left|\psi_{n}(t)\right|^{2}$ of the stationary bright solitonlike solution of the DNLS. Parameters are $\gamma=1, \mu=0$, and $V=0.2$. (b) The corresponding map plane depicting the homoclinic tangle of the hyperbolic fixed point at the origin. The amplitudes resulting from the dynamical study shown in (b) are shown as diamonds. They appear at the transversal intersections of the invariant manifolds.

stationary localized solutions to the DNLS related to homoclinic and heteroclinic orbits of the related map. This is the case even though there exist neighboring map orbits which are strongly chaotic. The reason is that the localized states rely on the structural stability of orbits homoclinic or heteroclinic to unstable hyperbolic fixed points such that their amplitudes are represented by a homoclinic (heteroclinic) orbit in the corresponding map plane of $\mathcal{M}$. A homoclinic point $\left(\phi_{n+1}^{h}, \phi_{n}^{h}\right) \equiv q$ is defined by $q \in \mathcal{W}^{s} \cap \mathcal{W}^{u}$ and $q=p$. Since $q$ belongs both to the stable and the unstable manifold of $p$ it follows that $\mathcal{M}^{n}(q) \rightarrow p$ as $n \rightarrow \pm \infty$.

In order to depict the homoclinic tangle of the global invariant manifolds we approximate the stable, respectively, the unstable, manifold in the vicinity of the hyperbolic fixed point by the linear subspaces (straight lines in the direction of the eigenvectors to the two eigenvalues with modulus apart from the unit circle) of the linearized map. Iterating a few thousand initial points on them several times, we obtain finally the homoclinic tangle of the hyperbolic fixed point. In
Fig. 4(b) we show the homoclinic tangle for the parameter choice of $\gamma=1, \omega=0.883$, and $V=0.2$. One clearly recognizes the homoclinic points. Points below the symmetry line $S_{0}$ are characterized by $\phi_{n+1}<\phi_{n}$ for $n>0$, i.e., they belong to $\mathcal{W}^{s}$. Each homoclinic point is mapped into another one and after only a few map iterations rapidly approaches the map origin where $\phi_{n} \rightarrow 0$.

Correspondingly, the homoclinic points above the line $S_{0}$ for which $\phi_{n}<\phi_{n+1}$ for $n>0$ will be mapped into the map origin in the course of the inverse map, i.e., they belong to $\mathcal{W}^{u}$, reflecting the translational invariance of the discrete lattice under the operation $n \leftrightarrow-n$.

Let us now use our knowledge about the homoclinic (heteroclinic) orbits to initiate (stationary) localized solutions for the time-dependent DNLS dynamics. In order to invoke the homoclinic map orbit as an initial condition for the dynamics, a sufficiently accurate location of the orbit members (homoclinic intersection points) is demanded. Obviously, the corresponding amplitudes could be read off from the map 
plane as the coordinates of the homoclinic intersections. However, this may not be accurate enough to ensure that the spatial behavior of the amplitudes of corresponding dynamical trajectory $\psi_{n}(t)=\phi_{n} \exp (-i \omega t)$ follows the homoclinic orbit $\phi_{n}^{h}$ closely enough, thus representing a nonlinear eigenstate. Therefore we use the normal form of Eqs. (56) and (58) to compute the homoclinic orbit "exactly."

For a study of the dynamics of solitonlike solutions for the DNLS given in Eq. (1) we use a lattice of chain length $N=201$. We implement the analytically computed homoclinic orbit $\phi_{n}^{h}$ with $n \in[-N / 2, N / 2]$ as initial conditions $\operatorname{Re} \psi_{n}(t=0)=\phi_{n}$ and $\operatorname{Im} \psi_{n}(t=0)=0$. The result for the solitonlike solution is illustrated in Fig. 4(a). Using a fastFourier-transform routine we determined the oscillation frequency to $\omega=0.879 \pm 0.004$, which is in fairly good agreement with the value for frequency put in the map, i.e., $\omega=0.883$. We inserted the (dynamical) amplitudes $\left|\psi_{n}(t)\right|^{2}$ as diamonds on the map plane in Fig. 4(b) to show that they coincide with the homoclinic orbit. The stationary localized solitonlike solution has the following amplitude pattern: $(\ldots, \uparrow, \uparrow, \uparrow, \ldots)$ where the dots stand for vanishingly small amplitudes. This localized mode is centered at a single site. Aceves and co-workers showed also that these excitation patterns of DNLS result in stable steady-state solutions [55-57].

In the same manner we proceed with the kinklike (dark soliton) solution for values of $\omega$ inside the linear band. To derive the heteroclinic orbit with high precision we apply a variational approach developed recently by Tabacman [49] to compute homoclinic and heteroclinic orbits for twist maps. The advantage of this method is that it only requires knowledge of the generating function of the map and a local approximation of the stable and unstable manifolds of orbits near the fixed points. The approximate manifolds can be obtained with the help of the linear subspaces of the tangent map taken at the fixed point at the origin as described above. Equipped with these approximate invariant manifolds, it remains to find the critical point of a certain function $W_{N}$ which is related to the generating function of the map (see Proposition 7 in [49]). The map can be rewritten in terms of the variables $q_{n}=\phi_{n}$ and $p_{n}=q_{n}-q_{n-1}$. The corresponding map orbits can be derived from the generating function

$$
\begin{aligned}
S\left(q_{n}, q_{n+1}\right)= & \frac{1}{2}\left(q_{n+1}-q_{n}\right)^{2}+\frac{1}{2 \mu}\left(\frac{\gamma}{\mu}-\omega\right) \ln \left(1+\mu q_{n}^{2}\right) \\
& -\left(\frac{\gamma}{2 \mu}+1\right),
\end{aligned}
$$

with the relations $p_{n}=-\partial S\left(q_{n}, q_{n+1}\right) / \partial q_{n}$ and $p_{n+1}$ $=\partial S\left(q_{n}, q_{n+1}\right) / \partial q_{n+1}$. One can define an action function $W_{N}$ the critical points of which deliver the orbit heteroclinic to the fixed points at $\left(\hat{q}_{-}=\hat{x}_{-}, \hat{p}_{-}=0\right)$ and $\left(\hat{q}_{+}=\hat{x}_{+}\right.$, $\left.\hat{p}_{+}=0\right)$. The action function $W_{N}$ is then given by

$$
W_{N}\left(q_{0}, \ldots, q_{N}\right)=\Phi^{u}\left(q_{0}\right)+\sum_{n=0}^{N} S\left(q_{n}, q_{n+1}\right)-\Phi^{s}\left(q_{N}\right),
$$

where the functions $\Phi^{u}\left(q_{0}\right)$ and $\Phi^{s}\left(q_{N}\right)$ describe locally the stable and unstable manifolds $\mathcal{W}^{u}\left(\hat{q}_{-}, \hat{p}\right)$ and $\mathcal{W}^{s}\left(\hat{q}_{+}, \hat{p}\right)$, respectively. These functions $\Phi^{s, u}$ can be computed using the linear subspaces at the fixed points. To compute the critical values of the function $W_{N}$ we used a Newton method. Apparently it is sufficient to obtain one single member of the heteroclinic orbit and then to use the map for getting the others as iterates. When iterating along the stable manifold we soon approach (typically after 5-8 numbers of iteration) the close vicinity of the hyperbolic fixed points where the orbit stays. Alternatively, one can also use normal form computations in order to generate the heteroclinic orbit. However, for heteroclinic orbits more than one normal form has to be evaluated.

Figure 5(a) shows the map plane for the kinklike solution. Again we have inserted the kink amplitudes $\left|\psi_{n}(t)\right|^{2}$ along the lattice as diamonds in the map plane shown in Fig. 5(b).

In this way excitation of the staggered solitons is also possible. Note that staggered localized DNLS modes have been observed experimentally in a real electrical network [58].

The map for the stationary solutions enables one also to predict the existence of another kind of stationary localized solution with amplitude pattern of the form $(\ldots, \uparrow, \uparrow, \uparrow, \uparrow, \ldots)$, i.e., it is supported by a homoclinic orbit having the turnstile as one homoclinic point located on the symmetry line $y=x$, i.e., $\phi_{n+1}=\phi_{n}$. This localized mode is centered between two lattice sites. Its energy is higher than that of the above mentioned localized mode centered at one single lattice site (see also next section).

We close this section with the remark that the complete dynamical DNLS system has also been studied [59]. It was found that the odd-parity mode is in fact a stable localized standing excitation of DNLS sustaining symmetry breaking perturbations of its mode pattern. Recently Aceves et al. also showed that the preferred stable localized DNLS states are supported by states having exponentially decaying amplitudes around the maximal amplitude at a single site, i.e., the odd-parity mode. On the other hand, the even-parity mode exhibits a dynamical instability and collapses to the oddparity mode under the impact of symmetry breaking perturbations. These results are in agreement with the findings in [60].

\section{THE SOLITON PINNING ENERGY}

As a consequence of the nonintegrability of the map $\mathcal{M}$ and the resulting transversal intersection of the stable and unstable manifolds the solitonlike solutions are pinned, i.e., they cannot be translated over the lattice from one point to an adjacent without overcoming an energetic barrier [29]. The pinning energy can be computed with the help of the normal forms as done in [52] for the solitons of the standard map. We use here another approach based on the findings of the Melnikov function. Kivshar and Campbell [25] studied the pinning energy (Peierls-Nabarro potential barrier) for the localized modes of the DNLS system, i.e., for $\gamma \neq 0$ and $\mu=0$.

There exist two homoclinic orbits whose points alternate 

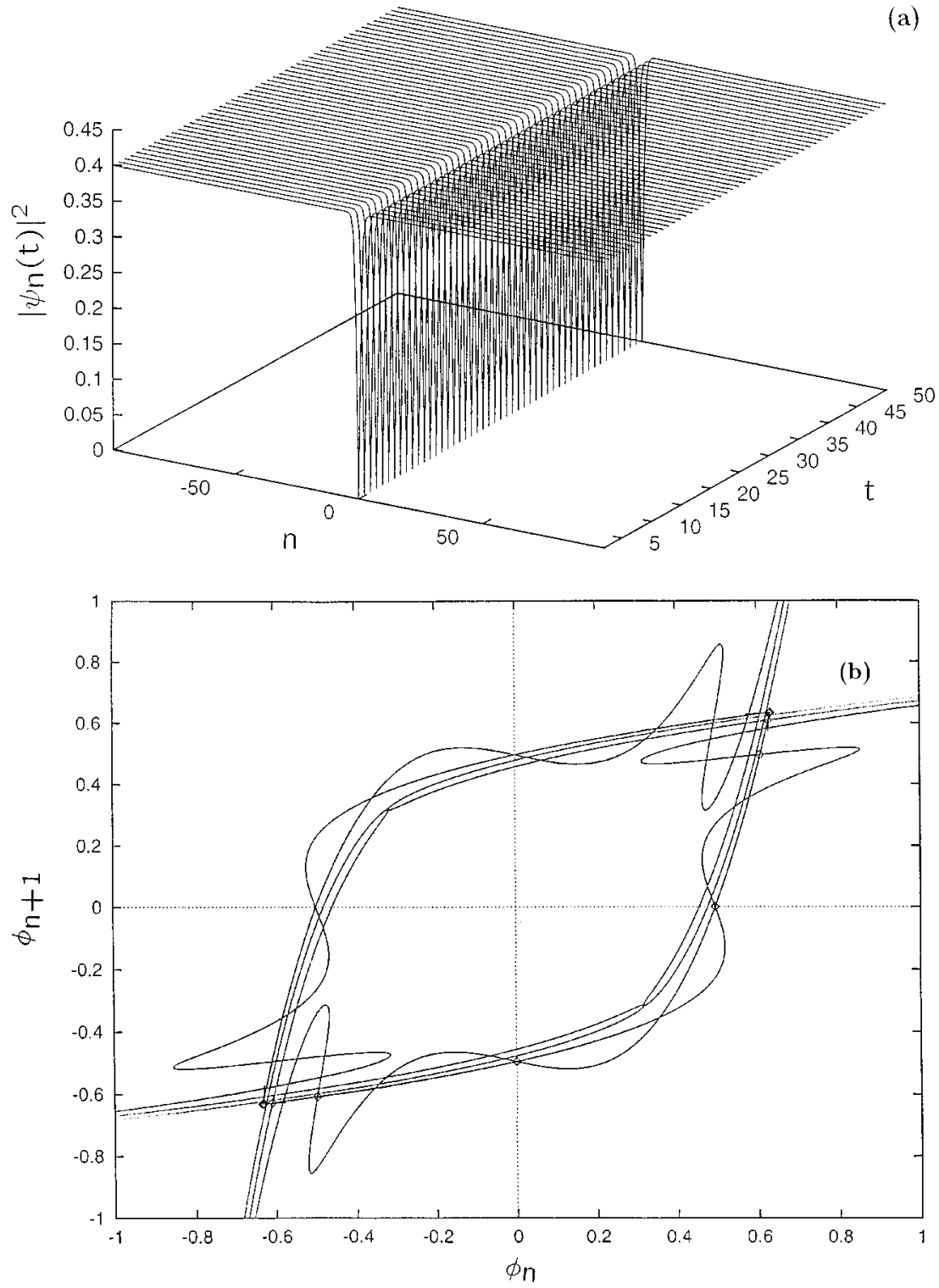

FIG. 5. (a) Profile $\left|\psi_{n}(t)\right|^{2}$ of the stationary kinklike solution (dark soliton) of the DNLS. Parameters as in Fig. 4. (b) The corresponding map plane illustrating the heteroclinic connection of the hyperbolic fixed points at $\left|\phi_{n+1}\right|=\left|\phi_{n}\right|$ $=\sqrt{-(\omega+2 V /) \gamma}$. The diamonds represent the squared modulus of the kink amplitudes taken from the dynamics of (a). along the invariant manifolds. Each of the homoclinic orbits has one of its points on the symmetry line $S_{0}$ and $S_{1}$, respectively. These points rapidly approach the map plane origin under the mapping where they stay most of the time. The homoclinic orbit crossing $S_{0}$ which we denote by $\left\{\Phi_{\text {even }}\right\}$ represents an excitation pattern of $\left.(\ldots \uparrow\rceil \uparrow \ldots\right)$ on the lattice chain. Such a stationary excitation pattern was called the even-parity mode in [3] and sometimes also the intersite centered local mode [60]. The other homoclinic orbit $\left\{\Phi_{\text {odd }}\right\}$ possesses three large amplitudes $\left(\phi_{-1}, \phi_{0}, \phi_{1}\right)$

and has the mode pattern $(\ldots \uparrow \uparrow \uparrow \ldots)$ which was called the odd-parity mode [2] or on-site centered local mode [60]. The point $\left(\phi_{-1}, \phi_{0}\right)$ is located on $S_{1}$. For positive (negative) $\gamma+2 \mu$ the unstaggered odd-parity (staggered even-parity) mode has lower action (energy) than the unstaggered evenparity (staggered odd-parity) mode. To see this for $\gamma+2 \mu>0$, one starts iterating the map $\mathcal{M}$ at the turnstile of $x_{\text {even }}^{\max }=y_{\text {even }}^{\max }$ (a member of the unstaggered even-parity mode), and goes along the stable manifold in the range of $y>x$ till the next intersection point is met. Then one follows the unstable manifold back to the turnstile. In this way a closed curve has been described and the area enclosed by it gives the action. We then apply the same procedure for the next pair of homoclinic points. Going down the stable manifold from the largest point of the unstaggered odd-parity mode $\left(x_{\text {odd }}^{\max }, y_{\text {odd }}^{\max }\right.$ ) one hits the next homoclinic point and then switches back to the unstable manifold. The obtained closed curve and thus the action (energy) is completely below the first one. Thus only the odd-parity map orbit corresponds to a physically relevant discommensuration of lowest energy. In the same manner one can show that for $\gamma+2 \mu<0$ the staggered even-parity mode has lower action (energy) than the staggered odd-parity one.

Following Aubry [29] we define the pinning energy as

$$
E_{p}=E_{\text {even }}-E_{\text {odd }} \text {. }
$$

The energy functional is given by 


$$
\begin{aligned}
E\left(\left\{\Phi_{n}\right\}\right)= & \sum_{n}\left[\frac{1}{2}\left(\phi_{n+1}-\phi_{n}\right)^{2}+\frac{1}{2 \mu}\left(\frac{\gamma}{\mu}-\omega\right) \ln \left(1+\mu \phi_{n}^{2}\right)\right. \\
& \left.-\left(\frac{\gamma}{2 \mu}+1\right) \phi_{n}^{2}\right] .
\end{aligned}
$$

We can compute $E_{p}$ " exactly" by using the homoclinic orbits obtained from the normal forms. Moreover, we can exploit the symmetry properties of the map $\mathcal{M}$. The Melnikov function provides us with the knowledge of the location of the intersections of the stable and unstable manifolds. Regarding the DNLS term proportional to $\gamma$ as a small perturbation to the AL map, we can get one orbit point for $\left\{\Phi_{\text {even }}\right\}$ as the intersection of the AL separatrix with $S_{0}$ as

$$
\phi_{-1}^{\text {even }}=\phi_{1}^{\text {even }}=\sqrt{-\frac{\omega+2}{\mu}} .
$$

To express the symmetry properties of the even-parity mode we take the lattice indices $n \in \mathcal{Z} \backslash\{0\}$. Similarly, we obtain for the point $\phi_{0}$ on $\left\{\Phi_{\text {odd }}\right\}$

$$
\phi_{0}^{\text {odd }}=\sqrt{\frac{1}{\mu}\left(\frac{\omega^{2}}{4}-1\right)} .
$$

The complete homoclinic orbits can be generated with help of the relations

$$
\begin{aligned}
\phi_{n}^{\text {odd }} & =\sqrt{\frac{1}{\mu}\left(\frac{\omega^{2}}{4}-1\right)} \operatorname{sech}[2 n \Delta t], \quad n=0, \pm 1, \ldots \\
\phi_{n}^{\text {even }} & =\sqrt{\frac{1}{\mu}\left(\frac{\omega^{2}}{4}-1\right)} \operatorname{sech}[(2 n+1) \Delta t], \quad n= \pm 2, \ldots .
\end{aligned}
$$

Using $\Delta t$ and $\phi_{ \pm 1}^{\text {even }}$ determined by (35) and (65), respectively, we obtain

$$
\begin{array}{r}
\phi_{n}^{\text {odd }}=\sqrt{\frac{1}{\mu}\left(\omega^{2}-4\right)\left(\lambda^{n}+\lambda^{-n}\right)^{-1}, \quad n=0, \pm 1, \ldots,} \\
\phi_{n}^{\text {even }}=\sqrt{\frac{1}{\mu}\left(\omega^{2}-4\right)\left(\lambda^{n / 2}+\lambda^{-n / 2}\right)^{-1}, \quad|n|>1 .}
\end{array}
$$

Taking the respective excitation patterns into account, we derive for the soliton energies

$$
\begin{aligned}
E_{\text {odd }}= & \frac{1}{\mu}\left[\omega^{2}-4\right] \sum_{n=0}^{N}\left[\left(\frac{1}{\lambda^{n+1}+\lambda^{-(n+1)}}-\frac{1}{\lambda^{n}+\lambda^{-n}}\right)^{2}-\left(\frac{\gamma}{\mu}+2\right) \frac{1}{\lambda^{n}+\lambda^{-n}}\right]+\frac{1}{\mu}\left(\frac{\gamma}{\mu}-\omega\right) \sum_{n=0}^{N} \ln \left(1+\left[\omega^{2}-4\right] \frac{1}{\lambda^{n}+\lambda^{-n}}\right) \\
& +O\left(\lambda^{-2(N+2)}\right)
\end{aligned}
$$

and

$$
\begin{aligned}
E_{\mathrm{even}}= & -\frac{1}{\mu} \sqrt{\omega+2}\left(\sqrt{2-\omega} \frac{1}{\lambda+\lambda^{-1}}-1\right)^{2}+\frac{1}{\mu}\left(\frac{\gamma}{\mu}-\omega\right) \ln \left[1+\mu\left(\phi_{1}^{\text {even }}\right)^{2}\right]-\left(\frac{\gamma}{\mu}+2\right)\left(\phi_{1}^{\text {even }}\right)^{2} \\
& +\frac{1}{\mu}\left[\omega^{2}-4\right] \sum_{n=0}^{2(N+1)}\left[\left(\frac{1}{\lambda^{(n+1) / 2}+\lambda^{-(n+1) / 2}}-\frac{1}{\lambda^{n / 2}+\lambda^{-n / 2}}\right)^{2}-\left(\frac{\gamma}{\mu}+2\right) \frac{1}{\lambda^{n / 2}+\lambda^{-n / 2}}\right]+\frac{1}{\mu}\left(\frac{\gamma}{\mu}-\omega\right) \\
& \times \sum_{n=0}^{2(N+1)} \ln \left(1+\left[\omega^{2}-4\right] \frac{1}{\lambda^{n / 2}+\lambda^{-n / 2}}\right)+O\left(\lambda^{-2(N+2)}\right) .
\end{aligned}
$$

A plot of the pinning energy as a function of $\omega$ is given in Fig. 6. The curve parameter is $\gamma$ and since $\gamma+2 \mu>0$ the pinning energy is positive. (Correspondingly, for negative $\gamma+2 \mu$ the pinning energy is negative.) The remarkable decrease of the pinning energy with increased $\gamma$ becomes clear in recalling that the computation of the pinning energy relied on the homoclinic orbit which was identified with location of the zeros of the Melnikov function on the unperturbed AL separatrix loop. This computation is the result of a perturbational calculation to first order in $\epsilon \gamma$. Moreover, the first correction to (66) is given by

$$
\phi_{0}^{\text {odd }}=\frac{1}{\gamma} \sqrt{\sqrt{\frac{4 \mu^{2}}{\gamma^{2}}+\left(\omega^{2}-4\right)}-2 \mu},
$$

demonstrating how the maximal amplitude of the odd-parity mode is shifted upwards on the AL separatrix loop with $\gamma$ diminishing the difference of the peaks of the odd-parity and even-parity modes. Finally the pinning energy decreases with increasing integrability parameter $\mu$.

We note that we can design an (unstaggered) odd-parity mode of desired width by varying $\omega$. If $\delta$ denotes a given decrease of the center amplitude then the lattice point $\widetilde{N}$ $\neq 0$ where $\phi_{\widetilde{N}}^{\text {odd }} \leqslant \delta \phi_{0}^{\text {odd }}$ holds becomes

$$
\widetilde{N} \geqslant\left[\frac{2}{\sqrt{\omega^{2}-4}-\omega} \cosh ^{-1}(\delta)\right],
$$




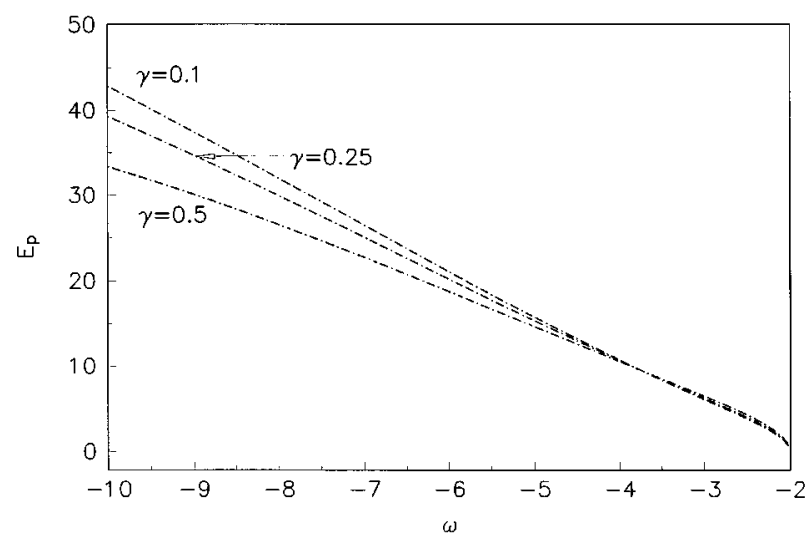

FIG. 6. Soliton pinning energy as a function of $\omega=-2.2 \mathrm{ob}-$ tained from Eqs. (71) and (72) with $N=64$ and $\mu=1$. Curve parameter is $\gamma$ as indicated.

where $[A]$ denotes the integer part of $A$. Similar expressions can be derived for the staggered odd-parity mode as well as the even-parity modes.

\section{SUMMARY}

We have studied in detail the stationary localized solutions of the GDNLS equation. First, we have described the general properties and features of the GDNLS and shown how this equation can be turned into a map by using a stationary solution ansatz. The bifurcational behavior of the fixed points of this map has been set out followed by a discussion of how the homoclinic and heteroclinic connections between the unstable fixed points can be related to the bright and dark solitons on the lattice. In Sec. III the DNLS term was assumed to be a small nonintegrable perturbation to the integrable $\mathrm{AL}$ equation, which allows us to calculate the Melnikov function explicitly. The latter describes the splitting of the separatrix related to the hyperbolic point at the map origin and leads to the result that the magnitude of the separatrix splitting depends exclusively on the ratio $\gamma /(\omega \mu)$. In investigations [33] this ratio was shown to determine the parameter region where the behavior of the map is regular. Furthermore, the Melnikov function shows that the position of the homoclinic intersections along the unperturbed homoclinic orbit solely depends on $\omega$ and not directly on the nonlinearity parameters $\gamma$ and $\mu$. In Sec. IV the Birkhoff normal forms were applied to calculate the homoclinic orbits related to the hyperbolic point at the map origin. The derived expression was shown to approximate the manifolds with high accuracy.

In Sec. V we have discussed how the homoclinic orbit of the related map supports localized solutions to the GDNLS.
This means that the irregular behavior of the map through the existence of homoclinic intersections actually ensures the existence of the localized solutions to the GDNLS. We also pointed out, in this way, that the map allows us to design localized excitation patterns of the GDNLS. Designing standing localized solutions of the GDNLS is only possible with the help of the stationary analysis which becomes clear from the fact that the broadness of a localized solution and its spatial exponential decay rate depend barely on the oscillation frequency $\omega$. The latter is accessible only in the stationary equation, whereas the two nonlinearity parameters $\gamma$ and $\mu$ appearing in the time-dependent GDNLS do not play a role for the purpose of soliton designing. Finally, we applied in Sec. VI the result of the Melnikov computations to calculate the pinning energy of the bright solitons on the lattice and showed that it can be tuned by varying the integrability and nonintegrability parameters, respectively.

It is interesting to compare the current findings with the result of Ref. [61] that continuous wave equations of the type $\square u=F(u)$ possess time-periodic and spatially localized solutions only for a small restricted class of functions $F(u)$. An example exhibiting time-periodic localized solutions is the completely integrable case of $F(u)= \pm \sin u$. In order to obtain solitonlike solutions of the field equations the authors of [61] used an asymptotic expansion method where the formal solution is represented in an asymptotic expansion as a power law of the leading nonlinear term. A base equation containing the essential nonlinearity is derived and the remaining hierarchy of equations is solved by a perturbation theory. The self-localized solution of the base equation is supported by a separatrix loop belonging to a hyperbolic point (the equilibrium state $u=0$ ) in the phase plane. It was shown that the dimension of the stable and unstable manifolds $\mathcal{W}^{s, u}$ of the hyperbolic point is, in general, finite. However, for localized solutions of the field system the existence of a separatrix loop with an infinite number of transversal intersections of $\mathcal{W}^{s, u}$ is demanded. Hence the infinite system of intersection conditions is overdetermined, which prevents the existence of time-periodic and spatially localized field solutions. Our approach of utilizing the separatrix intersections of a planar map to obtain solitonlike solutions of infinite lattice systems is successful, since the one-dimensional stable and unstable manifolds on the two-dimensional map plane inevitably intersect transversally as a result of the nonintegrability of the map.

\section{ACKNOWLEDGMENTS}

This work was supported by the Deutsche Forschungsgemeinschaft via Sonderforschungsbereich 337. One of the authors (K.Ø.R.) expresses his gratitude for the warm hospitality and financial support of the Institut für Theoretische Physik, Freie Universität Berlin.
[1] D.K. Campbell and M. Peyrard, in Chaos, edited by D.K. Campbell (AIP, New York, 1990), p. 305.

[2] A.J. Sievers and S. Takeno, Phys. Rev. Lett. 61, 970 (1988).

[3] J.B. Page, Phys. Rev. B 41, 7835 (1990).

[4] S. Takeno and S. Homma, J. Phys. Soc. Jpn. 60, 731 (1991);
62, 835 (1993); S. Takeno, ibid. 61, 2821 (1992); S.R. Bickham, S.A. Kiselev, and A.J. Sievers, Phys. Rev. B 47, 14206 (1993).

[5] T. Dauxois, M. Peyrard, and C.R. Willis, Physica D 57, 267 (1992); Phys. Rev. E 48, 4768 (1993); T. Dauxois and M. 
Peyrard, Phys. Rev. Lett. 70, 3935 (1993).

[6] Yu.S. Kivshar, Phys. Rev. E 48, 4132 (1993).

[7] S. Flach, C.R. Willis, and E. Olbrich, Phys. Rev. E 49, 836 (1994).

[8] S. Flach and C.R. Willis, Phys. Lett. A 181, 232 (1993); Phys. Rev. Lett. 72, 1777 (1994).

[9] Ch. Claude, Yu.S. Kivshar, K.H. Spatschek, and O. Kluth, Phys. Rev. B 47, 14228 (1992).

[10] S. Aubry, Physica D 86, 284 (1995).

[11] J.C. Eilbeck, P.S. Lomdahl, and A.C. Scott, Physica D 16, 318 (1985).

[12] V.M. Kenkre and D.K. Campbell, Phys. Rev. 34, 4595 (1986).

[13] A.S. Davydov and N.I. Kislukha, Phys. Status Solidi B 59, 465 (1973).

[14] N. Finlayson and G.I. Stegeman, Appl. Phys. Lett. 56, 2276 (1990)

[15] Y. Chen, A.W. Snyder, and D.J. Mitchell, Electron. Lett. 26, 77 (1990).

[16] F. Delyon, Y.-E. Levy, and B. Souillard, Phys. Rev. Lett. 57, 2010 (1986).

[17] M.I. Molina, W.D. Deering, and G.P. Tsironis, Physica D 66, 135 (1993).

[18] T. Holstein, Ann. Phys. (N.Y.) 8, 325 (1959).

[19] M. Toda, J. Phys. Soc. Jpn. 22, 431 (1975).

[20] M.J. Ablowitz and J.F. Ladik, J. Math. Phys. 17, 1011 (1976).

[21] R. Scharf and A.R. Bishop, Phys. Rev. A 43, 6535 (1991).

[22] B.M. Herbst and M.J. Ablowitz, Phys. Rev. Lett. 62, 2065 (1989).

[23] A.A. Vakhnenko and Yu.B. Gadidei, Theor. Math. Phys. 68, 873 (1986).

[24] K.W. Sandudsky, J.B. Page, and K.E. Schmidt, Phys. Rev. B 46, 6161 (1992).

[25] Yu.S. Kivshar and D.K. Campbell, Phys. Rev. E 48, 3077 (1993)

[26] M.J. Ablowitz and P. A. Clarkson, Solitons, Nonlinear Evolution Equations and Inverse Scattering (Cambridge University Press, New York, 1991).

[27] S. Takeno, J. Phys. Soc. Jpn. 58, 759 (1989).

[28] D. Cai, A.R. Bishop, and N. Grønbech-Jensen, Phys. Rev. Lett. 72, 591 (1994).

[29] S. Aubry, Physica D 71, 196 (1994).

[30] S. Aubry and G. Abramovici, Physica D 43, 199 (1990).

[31] R.S. MacKay and S. Aubry, Nonlinearity 7, 1623 (1994).

[32] P.C. Bressloff, Phys. Rev. Lett. 75, 962 (1995).

[33] D. Hennig, N.G. Sun, H. Gabriel, and G.P. Tsironis, Phys. Rev. E 52, 255 (1995).

[34] M. Salerno, Phys. Rev. A 46, 6856 (1992).
[35] J. Coste and J. Peyrard, Phys. Rev. B 39, 13086 (1989).

[36] Yi Wan and C.M. Soukoulis, Phys. Rev. A 41, 800 (1990); in Disorder and Nonlinearity, edited by A.R. Bishop, D.K. Campbell, and S. Pnevmatikos (Springer, New York, 1989), pp. 27-37.

[37] W. Chen and D.L. Mills, Phys. Rev. Lett. 58, 160 (1987); D.L. Mills and S.E. Trullinger, Phys. Rev. B 36, 947 (1987); H.G. Winful, Appl. Phys. Lett. 46, 527 (1985).

[38] Yu.S. Kivshar, Phys. Rev. Lett. 70, 3055 (1993).

[39] K.A. Ross and C.J. Thompson, Physica A 135, 551 (1986).

[40] G.R.W. Quispel, J.A.G. Roberts, and C.J. Thompson, Physica D 34, 183 (1989).

[41] G.R.W. Quispel, J.A.G. Roberts, and C.J. Thompson, Phys. Lett. A 126, 419 (1988).

[42] A.J. Lichtenberg and M.A. Lieberman, Regular and Stochastic Motion (Springer-Verlag, New York, 1992).

[43] J. Greene, J. Math. Phys. 20, 257 (1979).

[44] Yu.S. Kivshar and M. Salerno, Phys. Rev. E 49, 3543 (1994).

[45] R. Devaney, Trans. Am. Math. Soc. 218, 89 (1976).

[46] M.L. Glasser, V.G. Papageorgiou, and T.C. Bountis, SIAM J. Appl. Math. 49, 692 (1989).

[47] P.F. Byrd and M.D. Friedman, Handbook of Elliptic Integrals for Engineers and Scientists (Springer-Verlag, New York, 1971).

[48] G.D. Birkhoff, Acta Math. 43, 1 (1920).

[49] E. Tabacman, Physica D 85, 548 (1995).

[50] J. Moser, Commun. Pure Appl. Math. 9, 673 (1956).

[51] G.L. Da Silva Ritter, A.M. Ozorio de Almeida, and R. Douady, Physica D 29, 181 (1987).

[52] K. Furuya and A.M. Ozorio de Almeida, J. Phys. A 20, 6211 (1987).

[53] S. Aubry, Physica D 7, 240 (1983).

[54] S. Aubry and P.Y. Le Daeron, Physica D 8, 381 (1983).

[55] A.B. Aceves, C. De Angelis, A.R. Rubenchik, and S.K. Turytsin, Opt. Lett. 19, 329 (1994).

[56] A.B. Aceves, C. De Angelis, S. Trillo, and S. Wabnitz, Opt. Lett. 19, 332 (1994).

[57] A.B. Aceves, C. De Angelis, T. Peschel, R. Muschall, F. Lederer, S. Trillo, and S. Wabnitz, Phys. Rev. E 53, 1172 (1996).

[58] P. Marquié, J.M. Bilbault, and M. Remoissenet, Phys. Rev. E 51, 6127 (1995).

[59] D. Hennig (unpublished).

[60] E.W. Laedke, K.H. Spatschek, and S.K. Turitsyn, Phys. Rev. Lett. 73, 1055 (1994).

[61] V.M. Eleonskii, N.E. Kulagin, N.S. Novozhilova, and V.P. Silin, Theor. Math. Phys. 60, 395 (1984). 